Revista Mexicana de Economía y Finanzas Nueva Época

Volumen 14 Número 3, Julio-Septiembre 2019, pp. 433-446

DOI: https://doi.org/10.21919/remef.v14i3.353

\title{
La relación entre robo y desempleo de varones jóvenes en México, 2005-2017
}

Eduardo Loría

Universidad Nacional Autónoma de México, México

Emmanuel Salas ${ }^{1}$

Universidad Nacional Autónoma de México, México

(Recepción: 16/agosto/2017, aceptado: 16/enero/2018)

\section{Resumen}

A partir de la teoría de la elección racional probamos econométricamente los determinantes económicos del robo a través de un BVAR que analiza la relación del robo con la precariedad del mercado laboral y el desempleo de los varones de 15 a 24 años con baja escolaridad. Verificamos que existe un círculo vicioso entre precariedad del mercado laboral, robo, desempleo y actividad económica. Por lo tanto, sugerimos que una política de seguridad pública eficiente será aquella que no solo combata con firmeza la delincuencia, sino que también aborde su origen socioeconómico y reduzca la impunidad. La principal limitante es no considerar otros determinantes como el gasto en seguridad, pero la robustez de los resultados no indica que sea una carencia. Este artículo se destaca al modelar los determinantes del robo con este enfoque y no solo considerarlo una conducta antisocial. Es necesario articular una estrategia con varias acciones simultáneas que reduzcan rápidamente el robo porque encontramos que una vez que ha comenzado se reproduce.

Clasificación JEL: D01, C32, E24

Palabras clave: Robo Común, Condiciones Críticas de Ocupación, Desempleo Juvenil, lento crecimiento, VAR Bayesiano

\section{The relationship between theft and youth male unemployment in Mexico, 2005-2017}

\section{Abstract}

Using the theory of rational choice, we econometrically tested the economic determinants of theft through a BVAR that analyzes the relationship of theft with the precariousness of the labor market and the unemployment rate of men aged 15 to 24 years without higher education. We found that there is a vicious cycle between the precariousness of the labor market, theft, unemployment and economic activity. Therefore, we suggest that an efficient public security policy would be one that not only strongly fights crime but also simultaneously addresses its socioeconomic origin and reduces impunity. The main limitation is not considering other determinants such as spending on security, but the robustness of the results does not indicate that it is a deficiency. This article demonstrates that the determinants of theft can be explained by this approach and not just by antisocial behavior. It is necessary to articulate a multi-faceted strategy that could quickly reduce theft because otherwise, it will continue to proliferate.

JEL Classification: D01, C32, E24

Keywords: Common Theft, Critical Occupation Conditions, Youth Unemployment, Slow Growth, Bayesian VAR

\footnotetext{
${ }^{1}$ Centro de Modelística y Pronósticos Económicos (CEMPE), Facultad de Economía, UNAM. Agradecemos la asistencia en una versión anterior de Iván Ramírez. eduardol@unam.mx y salas.emmanuel@gmail.com teléfono 55562221 42. Agradecemos los valiosos comentarios de los árbitros de esta revista. Las aseveraciones, errores y omisiones que prevalezcan son de nuestra total responsabilidad. Este artículo es parte del proyecto de investigación Política monetaria y precarización del mercado laboral en México, una explicación alternativa al lento crecimiento 2000-2020 (IN300218), DGAPA, UNAM.
} 
REMEF (The Mexican Journal of Economics and Finance)

\section{Introducción}

La criminalidad se ha convertido quizá en el problema más serio en México por su alto impacto en el tejido social y en el crecimiento económico. La Encuesta Nacional sobre Victimización y Percepción de Seguridad Pública (INEGI, 2018a) reveló que el porcentaje de hogares con por lo menos un miembro afectado por la delincuencia ha subido sistemáticamente desde $32.4 \%$ en 2012 hasta $35.6 \%$ en 2017, lo que se traduce en 25.4 millones de víctimas mayores de 18 años. La misma encuesta informó que hubo 34 millones de delitos, de los cuales $28.1 \%$ fueron robos en la calle o transporte público. La encuesta estimó el costo total de la criminalidad en 2017 en alrededor de $1.65 \%$ del PIB. ${ }^{2}$

Por su parte, la Encuesta de Victimización a Empresas (ENVE) aplicada en 2017 (INEGI, 2018b) reportó que el $33.7 \%$ de los negocios fue víctima de algún delito y evalúa el costo que ha tenido la criminalidad para las empresas en alrededor de $1 \%$ del PIB ( $55.9 \%$ en pérdidas económicas y $44.1 \%$ en gasto en medidas preventivas ${ }^{3}$ ) y apunta a que el robo es el principal riesgo que enfrentan. También recoge las acciones que han tomado las empresas ante el crimen después de haber sido víctimas y todas tienen que ver con contracción económica, como son la reducción de horarios de operación (18.2\%), la cancelación de inversiones $(13.1 \%)$ y hasta el cierre de los negocios $(6.3 \%){ }^{4}$

Sin embargo, conviene recordar que la crisis de seguridad empezó mucho antes. Por eso fue que en 2006 se inició una estrategia que hasta ahora ha resultado fallida de combate al crimen. En efecto, a finales de ese año, el gobierno entrante definió una estrategia que a la fecha ha tenido resultados contrarios a los deseados, ya que el crimen, en todas sus manifestaciones, ha crecido mucho y muy rápidamente. Según el Informe Regional de Desarrollo Humano de PNUD (2013), con el inicio de la "guerra contra el narcotráfico" todos los delitos, incluyendo los de alto impacto como las tasas de homicidios y secuestros, crecieron aceleradamente (Molzahn et al., 2012) y en el año 2018 se rompieron récords históricos (SNSP, 2019).

La estrategia se centró en perseguir frontalmente al crimen organizado, castigarlo y no en prevenirlo. Lo anterior quizá derivó de la falta de un diagnóstico que asociara al crimen como un resultado del contexto socioeconómico y no solamente como una conducta antisocial aberrante. Tampoco se consideró que la oleada criminal podría responder a un serio problema de expectativas laborales caídas y, por tanto, de falta de movilidad social a pesar de que ha crecido la escolaridad promedio. Estos factores, que han sido dejados de lado en parte importante de la literatura han hecho que muchos jóvenes decidan delinquir como una manera de tener rápidamente mejores ingresos. Dentro de nuestro enfoque de racionalidad económica esto significaría que por las condiciones anteriores los agentes económicos en forma creciente han calculado que es mayor el costo de oportunidad de la educación.

Es importante comentar que fue tal la cantidad de abusos registrados en contra de las garantías individuales por esta estrategia, que en 2008 se hizo una importante reforma penal, de corte constitucional, que buscó respetarlas y no priorizar la encarcelación. Se argumentó que la cárcel lejos de ayudar a la reinserción social de los delincuentes, "mejoraba" sus habilidades y redes criminales, y los marginaba socialmente. Desafortunadamente, esta reforma penal por los resultados hasta ahora observados aumentó la impunidad derivada de las fallas en la actuación de la policía ${ }^{5}$ en el nuevo papel que le fue asignado, y

\footnotetext{
${ }^{2} \mathrm{~A}$ esto debemos sumar la falta en la cultura de la denuncia (que favorece el crimen) ya que INEGI (2018a) estima que la cifra negra (delitos sin denunciar) fue de $93.2 \%$ del total.

${ }^{3}$ Entre las más comunes están instalación o reemplazo de candados y cerraduras $28.9 \%$, reparación de puertas o ventanas $16.2 \%$, colocación de rejas o bardas $13.1 \%$, contratación de seguros $10.5 \%$ e instalación de seguridad contra ataques cibernéticos 5.9\%. (INEGI, 2018b).

${ }^{4} \mathrm{La}$ diferencia con el $100 \%$ se debe a acciones que no corresponden con la hipótesis del trabajo como: a) dejó de manejar efectivo y b) el dueño dejó de asistir.

${ }^{5}$ La policía no ha sabido llevar a cabo adecuadamente la aportación de pruebas que sustenten absoluta
} 
con ello la criminalidad.

Pero esta reforma tampoco consideró políticas de prevención efectivas. El Programa Nacional de Prevención del Delito (PRONAPRED), que se implementó en México de 2012 a 2016, se asoció a un aumento de la tasa de homicidios (Ramírez-de-Garay y Román, 2017) tal vez porque el programa incluía solo una cantidad limitada de acciones preventivas contra la proliferación de armas de fuego o sólo incluyó la resolución pacífica de conflictos en actividades como talleres de habilidades y/o valores o en campañas temáticas que no tuvieron gran impacto.

En este artículo solamente analizamos el robo $^{6}$ debido a que si bien no es calificado de alto impacto como el homicidio o el secuestro abre las puertas a otros de mayor impacto social y económico y porque ha mostrado un importante repunte desde 2016.Q2 y ha venido afectando notablemente la calma social, al patrimonio de la sociedad y a la actividad económica.

Lo anterior lo hacemos usando una línea de investigación que data al menos de los siglos XVII y XVIII con Beccaria y Bentham que establecieron el vínculo que existe entre el robo y la dinámica de los mercados laborales. Este artículo retoma este enfoque - pero rehabilitado por Becker (1976) - y considera al desempleo y a la tasa de Condiciones Críticas de Ocupación $(\mathrm{CCO})^{7}$ como variables fundamentales que inciden en el robo. A partir de la microfundamentación de estas variables podremos entender al crimen como una elección racional y no sólo como un atentado aleatorio producto de una conducta antisocial.

Nuestro enfoque plantea la presencia de un círculo vicioso dinámico que inicia con un choque negativo que eleva el desempleo de jóvenes varones con baja educación (hasta bachillerato), ${ }^{8}$ que en adelante mencionaremos como población objeto, $\mathrm{y} / \mathrm{o}$ con el aumento de $\mathrm{CCO}$ que precarizan el mercado laboral, afectando las expectativas de trayectoria laboral, de ingresos y bienestar futuros. Esto hace que - a partir de su cálculo racionalelijan al robo, lo que a su vez contrae la actividad económica.

Para dar una mayor fundamentación teórica a esta última relación, la Ley de Okun (1962) muestra que la elevación del desempleo y la precarización laboral sacrifican la actividad económica y a la inversión, tal como lo reportan para México Loría et al. (2015), y más recientemente con un enfoque de efectos no lineales asociados al ciclo económico Islas y Cortez (2018). En síntesis, un choque adverso deteriora las condiciones, trayectoria y expectativas laborales que a fin de cuentas aumentan el desempleo juvenil y este a su vez, deprime la actividad económica.

La preocupación por estudiar a los jóvenes se justifica al ver que las estadísticas muestran que la tasa de desempleo que los afecta es superior a la tasa de desempleo del resto de la economía. Tanto en el mundo como en México, la relación que guarda la tasa de desempleo juvenil (15-24 años) respecto de la de los adultos es alrededor del triple (ENOE, 2018). La ola de reformas económicas que se han aplicado, particularmente en los años recientes, no han logrado romper el círculo vicioso planteado en virtud de que la gran formalización del empleo se ha asociado a mayor precarización laboral.

e irrefutablemente la imputación del delito.

${ }^{6}$ En México el artículo 367 del Código Penal Federal define al robo como "el acto por el cual alguien se apodera de una cosa ajena mueble, sin derecho y sin consentimiento de la persona que puede disponer de ella con arreglo a la ley". En este artículo sólo analizamos los robos denunciados (tasa) ante el Ministerio Público expresada en eventos por cada 100,000 habitantes. No incluimos otras quejas o acusaciones y no distinguimos entre diferentes tipos de robo ni lugar o espacio donde fueron cometidos.

7"Personas que trabajan menos de 35 horas a la semana por razones ajenas a sus decisiones, más las que trabajan más de 35 horas semanales con ingresos mensuales inferiores a un salario mínimo y las que laboran más de 48 horas semanales ganando hasta dos salarios mínimos" (STPS, 2017).

${ }^{8}$ Definimos como baja educación hasta bachillerato porque el artículo 3ro de la Constitución Política de los Estados Unidos Mexicanos refiere que la educación media superior, bachillerato, será la máxima obligatoria. Por ende, consideraremos baja educación a cualquier grado igual o inferior a la educación obligatoria por ley. 
El artículo, además de esta introducción, presenta una revisión de literatura, un enfoque teórico que microfundamenta la elección del robo, aspectos econométricos y las conclusiones que incluyen recomendaciones de política.

\section{Revisión de literatura.}

Desde la perspectiva de Becker (1976) las personas asignan su tiempo en función de la actividad que más beneficios netos les reporte, de manera que toda elección de actividad es resultado de un cálculo de beneficios y costos. Al plantear que es una elección racional, es plausible pensar que existe un sistema de incentivos y desincentivos que permite abordar el estudio del robo desde la economía y no sólo desde otras disciplinas como el derecho y la psicología (Becker, 1976).

En este sentido, este artículo recupera lo que Cantor y Land (1985) llaman "efecto de motivación criminal" que refiere que la precariedad económica (laboral, de ingresos y la afectación de las expectativas) induce a los individuos a incurrir en actividades delictivas. Como ejemplo, Mendoza (2013) reporta que por el súbito incremento del desempleo a razón de la crisis del 2009, la tasa de delitos (eventos registrados por cada 100,000 habitantes) en la frontera norte de México aumentó alrededor del $10 \%$.

Las condiciones familiares son otro factor muy importante para cometer actos criminales, principalmente robos. De acuerdo con Varella (2014), las conductas agresivas y antisociales de la población objeto son resultados del estrés familiar ${ }^{9}$ que conducen a este grupo de población a tomar decisiones riesgosas, ya que no existe una preocupación por las consecuencias de sus actos delictivos (Obando y Ruiz, 2007). Freeman (1999) explica que los criminales tienden a ser jóvenes varones menos educados que provienen de hogares problemáticos y de minorías, debido a su reducida expectativa de tener altos ingresos legítimos en el largo plazo y de tener alto resentimiento social. Al respecto, desde el ámbito del derecho, De Gante (2017) comenta que la razón principal del crimen es de carácter estructural vinculado con la marginación, la pobreza y la crisis de expectativas.

Respecto de los jóvenes y su ambiente social Zuluaga et al. (2018) señalan que existe una fuerte relación entre desempleo y violencia en los jóvenes. Por lo anterior, es que existen iniciativas que buscan desincentivar la violencia a través de la creación de empleo y oportunidades económicas.

Moreno y Toledo (2017:5) reportan el alto reclutamiento de jóvenes por el crimen organizado, que coincide con el estudio del Banco Mundial (2013) que reporta que más de la mitad de los delitos en México durante 2010 se cometieron por jóvenes, de los cuales la mayoría tenía entre 18 y 24 años y 9 de cada 10 eran hombres, lo que también coincide con lo reportado por Thornberry y Christenson (1984) quienes encontraron que el desempleo y las condiciones sociales tienen efectos significativos sobre el crimen, en especial entre jóvenes afroamericanos y de bajos estratos sociales, blue-collar backgrounds.

Gómez y Merino (2012) estudiaron la deserción escolar de los jóvenes como un factor que impulsa su desocupación laboral o su precaria incorporación al mercado laboral y su consecuente inclusión en grupos delictivos. La educación representa tiempo a invertir con el fin de generar mayores ingresos futuros; sin embargo, para el caso de los jóvenes que no estudian ni trabajan la expectativa de ingresos futuros por estudiar es baja, por lo cual prefieren dedicarse a la delincuencia. Es decir, su costo de oportunidad de realizar estudios largos es muy alto en relación con los beneficios económicos de insertarse al crimen y así obtener ingresos rápidos y altos.

Con el antecedente del Banco Mundial (2013), Fajnzylber et al. (1998) y Roemer (2001) concuerdan que el sexo y la edad son factores determinantes para delinquir. Por

\footnotetext{
${ }^{9}$ Arias et al. (2010) definen al estrés familiar como condiciones socioeconómicas, de crianza y culturales adversas que son resultado de familias monoparentales crecientemente encabezadas por mujeres y madres solteras que por su misma condición determina poca atención y cuidado de los hijos, y ello se convierte en un factor de prevalencia.
} 
todo lo anterior, es que estimamos razonable centrar nuestra atención en los jóvenes varones con baja educación y en condiciones de precariedad laboral, porque es el grupo que potencialmente tiene más proclividad a cometer ilícitos. Lo anterior no implica criminalizar a todo este grupo sociodemográfico. Pero es el que presenta la mayor condicionalidad de prevalencia.

Además de las condiciones anteriores, existen otras que también son de suma importancia como las asociadas a la falta de castigo, que pueden ser aún más importantes para elegir esta actividad. A ese respecto Beccaria (1764) afirma que el aumento de la probabilidad de aprehensión es crucial para prevenir los delitos en el corto plazo: "Lo que más disuade a los ciudadanos de violar la ley no es la exagerada gravedad de la pena, sino la inexorabilidad de la justicia. No se deben aplicar castigos inhumanos, sino aplicar castigos relativamente leves pero con toda seguridad".

Urrusti (2012) reporta que para México la falta de coordinación entre el gobierno federal y los gobiernos estatales eleva la criminalidad y la violencia, debido a que reduce la probabilidad de captura y procesamiento penal de los criminales y con ello el costo de delinquir. A lo anterior debemos adicionar que hay carencias graves en la capacitación de los policías mexicanos que han abierto gran espacio de impunidad ya que no han sido capaces de ejecutar con solvencia el registro de pruebas que permita la consignación y el adecuado proceso del indiciado (Aguirre, 2018).

Asimismo, la reforma constitucional en materia penal de 2008 que cambió el modelo penal ${ }^{10}$ que incorpora más medidas de protección, precautorias y cautelares que no necesariamente implican la privación de la libertad del imputado, crea un mecanismo alternativo de la solución de controversias y también asigna más responsabilidades a la policía para hacer una adecuada consignación.

Esta reforma buscó fomentar los procesos penales en libertad ya que en México, debido al rezago penal, $40 \%$ de la población carcelaria no cuenta con una sentencia (de culpabilidad o de inocencia) y en muchos casos ha resultado que tras varios años de estar encarcelados no se les puede comprobar culpabilidad (Arévalo, 2016:5).

Es por ello que el nuevo sistema penal ha preponderado la presunción de inocencia y evitado medidas de encarcelamiento a menos que la policía aporte la evidencia suficiente y cumpla con el debido proceso para que el juez imponga encarcelamiento precautorio.

La justificación de esta reforma la encontramos en Cid (2007) quien presenta dos visiones respecto de las penas en prisión. La primera, conocida como de "prevención espacial negativa", menciona que las penas privativas de la libertad son más eficientes para evitar la reincidencia porque crea un efecto de supresión que se basa en el supuesto de que una persona que ha sufrido la pena de cárcel tendrá más aversión a cometer un crimen. La segunda, la teoría del "etiquetamiento", considera que los procesos privativos de la libertad son criminógenos y predisponen a la reincidencia debido a que el proceso de encarcelamiento degrada a las personas y hace surgir en ellas formas alternativas de socialización en sub-culturas criminales. Además, el encarcelamiento crea dentro de la persona un sentimiento de injusticia y de venganza que se reforzará cuando advierta que su condición de ex recluso le complique la reinserción social.

\section{Aspectos teóricos.}

A partir del análisis de la literatura y de la motivación del artículo, proponemos que el aumento del desempleo de la población objeto y de personas en CCO es un factor de incidencia de robo. Asimismo, la combinación de las condiciones de ineficiencia de la policía y del poder judicial junto con las condiciones carcelarias que prevalecen en México hacen que el robo se auto reproduzca, a manera de un proceso de histéresis.

Para formalizar este enfoque definimos la utilidad neta de la realización o no de una

${ }^{10}$ De uno inquisitivo a uno basado en juicios orales, De Gante (2017). 
actividad, que será el producto del beneficio obtenido por su ejercicio, ${ }^{11}$ descontada la desutilidad que refiere al costo (riesgo) de hacerlo (Becker, 1976).

Nos enfrentamos, de entrada, con un problema de unidades de medida, ya que los beneficios del robo se miden en unidades monetarias, mientras que los riesgos físicos, la probabilidad de ser capturado y castigado y el tiempo invertido se expresan en otras unidades difícilmente medibles en esos términos, por lo que analizamos a la elección racional a través del axioma débil de la preferencia revelada (Mas-Colell et al., 2006:10) que permite aseverar que si un agente racional tiene un orden de preferencias, sus decisiones generarán acciones que necesariamente reflejarán sus preferencias, que implica que si una cesta $x$ es elegida en la presencia de y, no existirá una cesta que conteniendo ambas alternativas y sea preferido a x (Mas-Colell et al., 2006:12). En síntesis, la elección de una actividad particular como el robo revela una preferencia, que es racional con lo que implícitamente resuelve el problema de optimización y de unidades de medida.

De esta manera, los agentes eligen la actividad que maximiza su beneficio neto al evaluar los ingresos probables de la actividad y la desutilidad en la que incurren, dado su sistema de valores personales y aspiraciones de corto, mediano y largo plazos.

Así, el beneficio neto de emplearse en una actividad legal $\left(B n_{E}\right)$ está determinado por el salario de mercado $\left(w_{m}\right)$ menos la desutilidad del trabajo que representa renunciar al ocio. Nuestro análisis considera que, por las consideraciones ya descritas de baja calificación educativa y laboral la población objeto se encuentra en condiciones de gran estrés laboral, por lo que es plausible aceptar que su utilidad del trabajo cae sistemáticamente a una tasa $\mathrm{k}$ en el tiempo. Por otro lado, también pueden enfrentar alto estrés familiar en la medida que deben convertirse en proveedores desde edad muy temprana. Todo ello hace que el costo de oportunidad de permanecer empleado en esas condiciones aumente rápidamente con el paso del tiempo. La ecuación (1) sintetiza a este primer grupo de selección:

$$
B n_{E}(t)=w_{m}+s e^{-k t}
$$

Es decir, con el tiempo la satisfacción (s) de mantenerse en CCO cae a la tasa $k$, hasta que sólo queda como beneficio neto el componente salarial.

Por su parte, el beneficio neto del desempleo $\left(B n_{U}\right)$ captura el hecho de que el ocio genera satisfacción (A) que disminuye en el tiempo a la tasa h. Así como en el caso del empleo precario, esa utilidad decae a la tasa h debido al costo de oportunidad que representa no tener ingresos:

$$
B n_{U}(t)=c+A e^{-h t}
$$

donde $c$ es el beneficio máximo que puede tener un individuo desempleado.

Por último, y a diferencia de los dos casos anteriores, el beneficio neto del robo tiene una trayectoria creciente, porque es plausible considerar que el ingreso total y marginal de un ladrón aumenta con cada robo porque también lo hace el valor del botín debido a que también crece la habilidad con la práctica y a que vende el botín a un precio cada vez mayor porque su experiencia en el mercado negro también crece. Asimismo, es razonable suponer que la desutilidad del robo (el costo asociado al esfuerzo y al peligro, pero sobre todo a la reducción de la probabilidad de captura y condena) cae con cada crimen. Por lo tanto, a diferencia de las otras dos actividades, el beneficio neto en el tiempo aumenta en un factor $g$ :

$$
B n_{r}(t)=B e^{g t}
$$

De lo anterior no podemos decir que toda la población objeto se dedicará al robo. Para que ello ocurra será necesario que, en un punto, los beneficios de las opciones legales $(i)$,

\footnotetext{
${ }^{11}$ Esta definición excluye la satisfacción u otros factores adicionales que pueden actuar en favor de la elección.
} 
Empleo (E) o Desempleo (u), sean inferiores a los del robo, tal como se expresa en (4):

$$
B n_{r}(t)>B n_{i}(t), \quad i=E, u
$$

Como contra ejemplo, existen actividades que también reportan ingresos bajos y ponen en riesgo la vida como el ser soldado o policía en las que también se emplean muchos jóvenes varones. Pero la aceptabilidad y reconocimiento social que les reporta eleva su satisfacción, que equivaldría a una compensación extra económica $(s)$ como se expresa en la ecuación (1), y esto hace que no se dediquen al crimen.

Con base en lo anterior, en la figura 1 proponemos la presencia de un círculo vicioso entre CCO, tasa de desempleo de la población objeto y lento crecimiento del PIB. Esta última variable se sustenta teóricamente a partir de la Ley de Okun (1962), que describe una fuerte relación bidireccional negativa entre la tasa de desempleo y el crecimiento económico.

Comúnmente la relación se ha analizado del producto al desempleo; sin embargo, varios trabajos como el de Prachowny (1993) y Dornbusch et al. $(2004: 132)^{12}$ prueban y evalúan el efecto contrario; es decir, del desempleo al producto.

Esta ley ha dado pie a una prolífica literatura empírica, y para México sus efectos se han probado recientemente - entre otros - por Loría et al (2015) e Islas y Cortez (2018).

Este círculo vicioso, eminentemente dinámico, comienza a partir de un choque económico que eleva la precariedad laboral y/o el desempleo. En cualquier caso, y por los elementos teóricos ya expuestos, la circularidad se activa afectando eventualmente a todas las variables involucradas.

En el apartado siguiente se hace la estimación econométrica de esta circularidad. No es posible introducir simultáneamente a la tasa de desempleo y al PIB en virtud de que son altamente colineales, por lo que incluso el sistema no puede resolverse debido a la dependencia lineal casi perfecta. Para corroborarlo estimamos el factor de inflación de varianza (VIF) como lo plantean Zuur et al. (2010) y el valor es de 97.77 lo que corrobora fuerte colinealidad que impide estimar el BVAR.

Con el propósito de no perder riqueza analítica, y por lo que supone la Ley de Okun, es preferible incorporar solamente a la tasa de desempleo. Sin embargo, si incluimos al PIB en lugar de la tasa de desempleo, la inferencia estadística no se modifica.

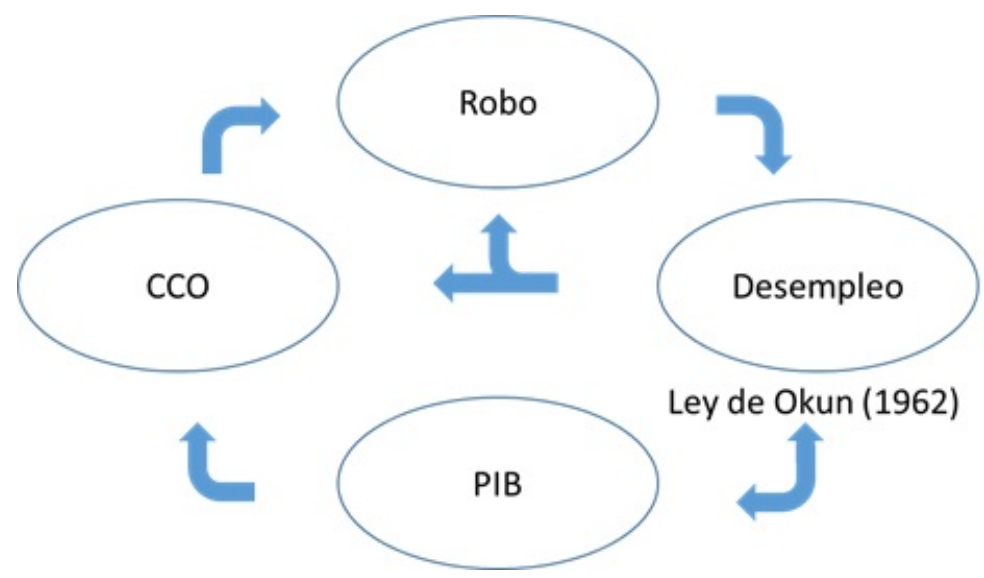

Figura 1. Círculo vicioso de robo, precariedad laboral, desempleo y lento crecimiento Fuente: elaboración propia.

${ }^{12}$ Estos autores reportan que el aumento de $1 \%$ de la tasa de desempleo sacrifica $2 \%$ del PIB. 


\section{Aspectos econométricos}

Los variables utilizadas fueron la tasa de robos (eventos denunciados por cada 100,000 habitantes, SNSP, 2019), Tasa de Desempleo de la población objeto ${ }^{13}$ (ENOE, 2018) y la Tasa de Condiciones Críticas de Ocupación, CCO (ENOE, 2018). Se ocupó la muestra más amplia disponible, que arranca desde 2005.1.

En la gráfica 1, podemos apreciar un conjunto de hechos estilizados muy importantes en términos de nuestro objetivo: a) la tasa de robos aumentó vertiginosamente hasta 2011, muy probablemente como consecuencia de los estragos de la Gran Recesión. A partir de ese año, la lenta recuperación económica redujo la tasa de desempleo y la incidencia del delito, b) posteriormente la tasa de robos repuntó rápidamente desde 2016, justo cuando la reforma penal inició su aplicación ${ }^{14}$ y c) es precisamente después de 2015 , en que la tasa de desempleo comenzó a caer cuando CCO comenzó a crecer notablemente. Esto estaría indicando que la recuperación económica y la generación de empleos formales se han dado a partir de la precarización laboral lo cual apoya la hipótesis central del artículo.

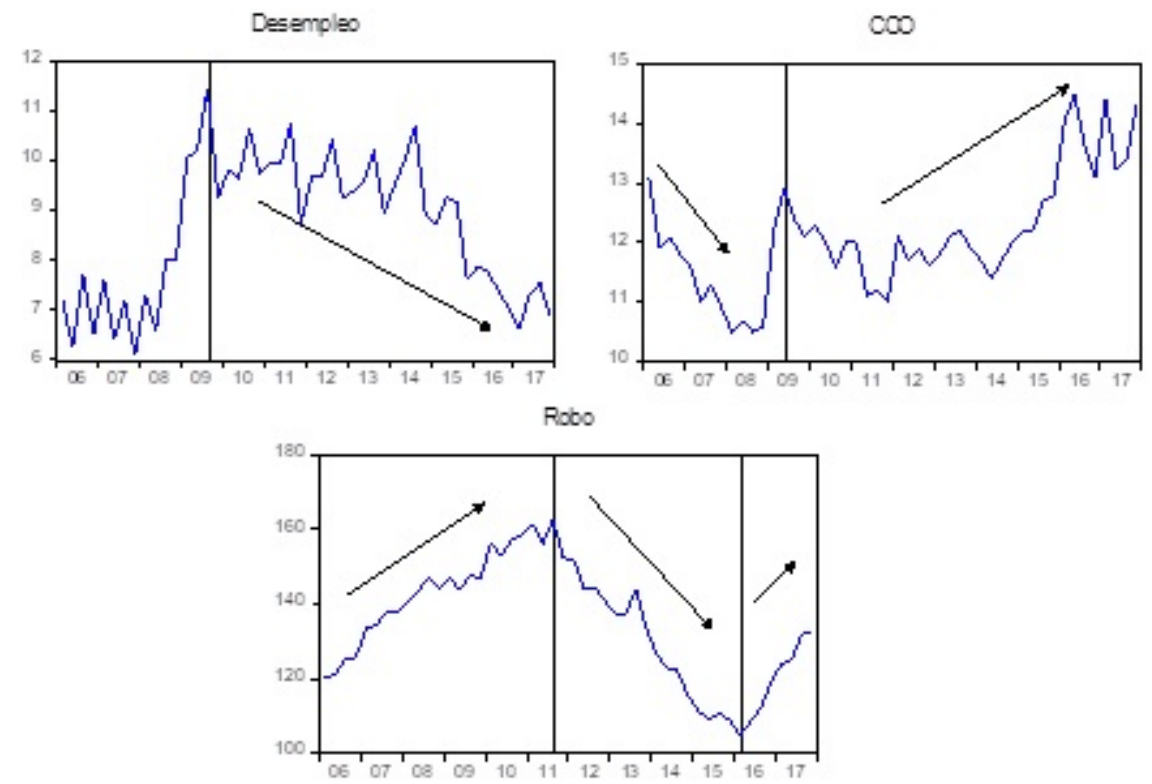

Gráfica 1. Variables del modelo Fuente: ENOE (2018) Y SNSP (2019).

Con la finalidad de probar empíricamente nuestra hipótesis y así captar los múltiples efectos entre las variables seleccionadas estimamos un sistema de ecuaciones a partir de un modelo BVAR. En los modelos VAR clásicos, aún de dimensión reducida, existe el problema de la gran cantidad de parámetros a estimar. Así, por ejemplo, para un VAR (3) con 4 variables será necesario estimar 52 parámetros, lo que hace que se agoten rápidamente los grados de libertad, lo que a su vez lleva a una situación en la que cada punto está "explicado" por un parámetro como mínimo, de manera que el modelo es incapaz de discriminar adecuadamente los elementos sistemáticos y los puramente accidentales que subyacen en las observaciones (Quilis, 2002:17).

Esta particularidad hace que sea cuestionable la precisión y la inferencia por el problema recurrente de multicolinealidad, que tiene por consecuencia el aumento de las varianzas

\footnotetext{
${ }^{13} \mathrm{La}$ tasa de desempleo de los jóvenes varones con baja educación se calculó de un filtrado de datos de la ENOE (2018) a partir de la imposición de 3 condiciones: a) edad entre 15 y 24 años, b) sexo masculino, c) escolaridad máxima de bachillerato. Ese número de desempleados se dividió entre la población económicamente activa (ENOE, 2018). Agradecemos el apoyo de Iván Ramírez para este cálculo.

${ }^{14} \mathrm{Si}$ bien su aprobación fue en 2008, su ejecución inició en 2016.
} 
de los estimadores, además de problemas estadísticos inherentes a su propia construcción. ${ }^{15}$

El enfoque bayesiano sugiere una aproximación diferente para superar estos problemas al minimizar la cantidad de rezagos, mediante la especificación de un conjunto de hipótesis a priori que suplementan los datos. En esta línea de análisis, Litterman (1984) plantea que la dinámica propia de cada variable es más importante que la cruzada, que la influencia disminuye a medida que aumenta la distancia temporal, que las series individuales pueden o no son ser estacionarias y puede o no existir cointegración. ${ }^{16}$

Los modelos BVAR hacen análisis inferencial a través de las funciones impulso-respuesta (Quilis, 2002: 29 y Lu et al, 2013). Para probar la hipótesis central del artículo estimamos un BVAR con prior estructura de Normal-Wishart ${ }^{17}$ para parámetros de forma reducida $\left(\alpha, \sum e\right)$, (Canova, 2007: 369) con un estimador $\bar{\theta}=\bar{V}\left[V_{0}^{-1} \theta_{0}+X X \hat{\theta}\right.$ y una matriz de varianzas y covarianzas $\bar{V}=\left[V_{0}^{-1}+X X\right]^{-1}$.

Ambas expresiones conjugan el prior con la información muestral. Siguiendo el proceso de calibración propuesto por Quilis (2002) se impusieron los siguientes valores para los hiperparámetros $i \mu_{1}=0, \lambda_{1}=0,1$, (Kadiyala y Karlsson, 1997). ${ }^{18}$

Para las gráficas 2, 3 y 4 las bandas de confianza se calcularon con el método de Bootstrap (método percentil) con 1000 iteraciones que permite obtener la desviación estándar de la muestra al $95 \%$ de confianza, a partir de los cálculos de la desviación estándar de Singh y Xie (2008). Se utilizó el método de impulsos generalizados para evitar la sensibilidad al ordenamiento del sistema (Pesaran y Shin, 1998). ${ }^{19}$

En la gráfica 2 se aprecia que la respuesta de los robos a sí mismos indica histéresis. Adicionalmente, es de destacar que es la mayor respuesta cuantitativa de todo el modelo (empieza en 4.5 y después de veinte periodos, se mantiene en 3.5). Esperábamos este resultado a partir de la formalización que hicimos en la sección anterior. Llama la atención que el robo se vuelve significativo al desempleo después de ocho trimestres, lo cual se inserta claramente en la hipótesis de que con el tiempo la desutilidad de mantenerse en esa situación crece por lo que los individuos elegirán al robo como opción de ingresos. Es notable, por otro lado, que CCO tiene un efecto casi inmediato sobre el robo, lo que sugiere que el costo de oportunidad de los jóvenes de mantenerse en condiciones precarias de ocupación es muy alto, por lo que, rápidamente pueden elegir al robo como opción de ingresos.

\footnotetext{
${ }^{15}$ Quilis (2002:12) explica que cada variable en un VAR es generada a partir de la suma algebraica de tres elementos: valores desfasados de la propia variable (dinámica propia), valores desfasados de las restantes variables (dinámica cruzada) y las innovaciones específicas.

${ }^{16}$ Desde el prior Minessota-Litterman, puede existir la presunción de que las series evolucionan a partir de la presencia de una raíz unitaria (Litterman, 1984).

${ }^{17}$ Que suprime el supuesto restrictivo del prior de Minessotta-Litterman sobre el conocimiento previo de la estructura de la matriz de varianzas y covarianzas.

${ }^{18}$ Los resultados con otros valores en los hiper parámetros no varían significativamente en términos de la validez de la hipótesis.

${ }^{19}$ Aunque con el ordenamiento de Cholesky no detectamos cambios substanciales.
} 

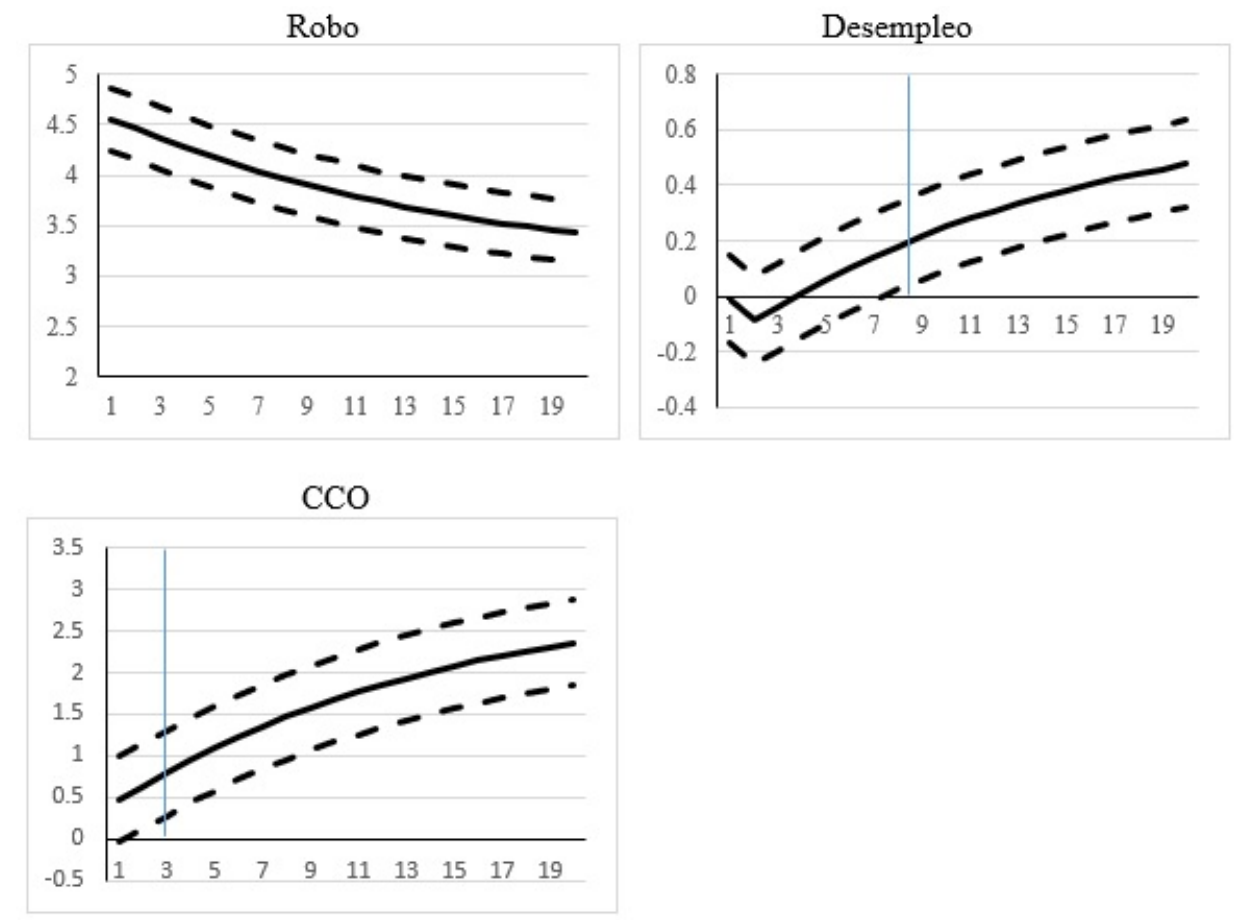

Gráfica 1. Respuestas del robo a:

Por su parte, la gráfica 3 muestra hechos muy importantes en términos de la hipótesis. Por un lado, encontramos que la respuesta del desempleo a los choques de sí mismo no es permanente, ya que el impulso es estadísticamente diferente de cero solo en los primeros 2 periodos, lo que implica que los choques de la tasa de desempleo de la población objeto no se auto reproducen, con lo cual se sugeriría que salen del mercado laboral o se insertan rápidamente en las opciones de ocupación aquí descritas.

Por otro lado, es muy notable el efecto positivo, significativo y permanente del robo sobre el desempleo lo que demuestra fehacientemente el hecho de que la criminalidad afecta de inmediato a la actividad económica y, por lo tanto, reduce las condiciones de empleabilidad de los jóvenes. ${ }^{20}$ La población en general reduce sus consumos y sus hábitos de salir por las noches - incluso durante el día en bastas zonas del país - y los negocios reducen sus horarios de operación ante el aumento de los asaltos y robos.

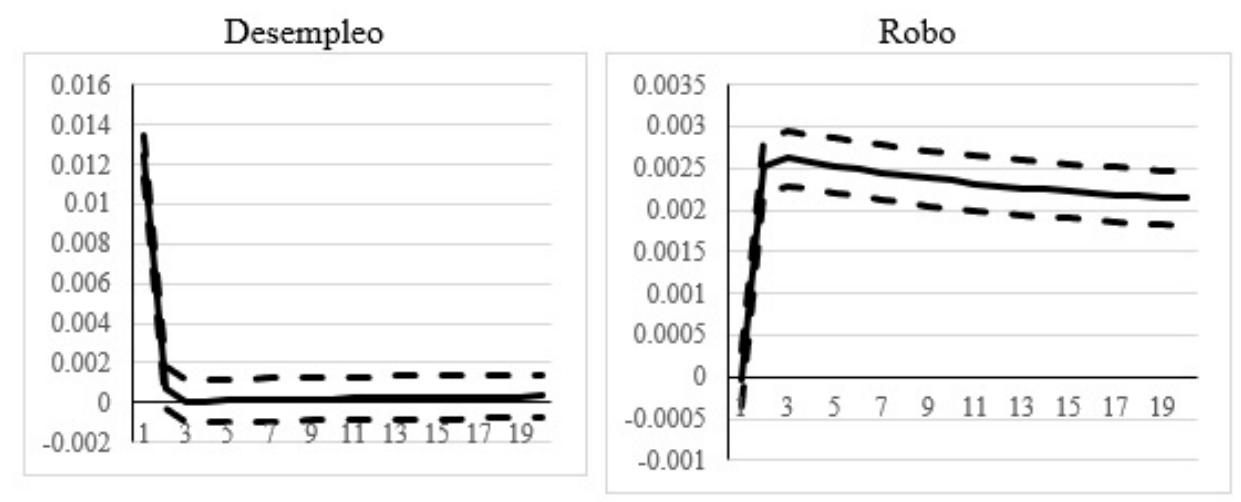

Gráfica 3. Respuesta del desempleo a:

\footnotetext{
${ }^{20}$ Este efecto prueba empírica y fehacientemente la operatividad de la Ley de Okun.
} 
Por último, la gráfica 4 permite redondear y fortalecer los resultados anteriores a partir de la dinámica de $\mathrm{CCO}$. En primer lugar observamos que $\mathrm{CCO}$ reacciona positivamente a sí misma con presencia de histéresis; lo que nos parece muy preocupante, ya que no importa si el estímulo inicial que motivó el crecimiento de CCO ya no está presente, esta variable se mantendrá en niveles altos y, como vimos en la gráfica 2, además de mantener la precariedad laboral, impulsará al robo, lo que crea el círculo vicioso ya descrito.
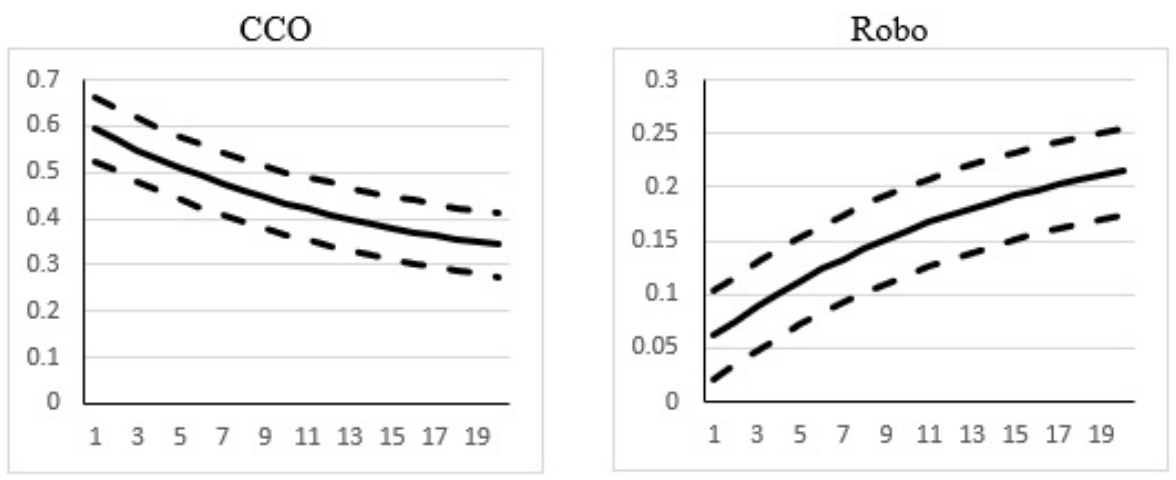

Gráfica 4. Respuesta de CCO a:

\section{Conclusiones.}

En este artículo analizamos las condiciones socioeconómicas del robo en México, en el periodo más largo que las estadísticas lo permitieron. A partir de la revisión de la literatura y de la estimación econométrica sugerimos que el robo es una elección racional que se da en un entorno de precariedad social y económica y de crisis de expectativas de los retornos de la educación y, por tanto, de las trayectorias laborales. Al respecto, Infante et al. (2010) mencionan que un bajo nivel de educación es una característica importante de las personas que cometen robos.

Por lo anterior, construimos un enfoque teórico microfundamentado que establece la racionalidad de la elección del estado de ocupación, en específico, del robo, que permite entender que elegirlo como actividad no es sólo una anomalía conductual.

Adicionalmente, el INEGI (2018a y 2018b) ha reportado que el robo tiene un efecto contractivo en el PIB, tanto por las reacciones de las personas como de las empresas afectadas ${ }^{21}$ lo que incrementa el desempleo y la precariedad del mercado laboral. De hecho, el $63 \%$ de las unidades económicas consideran que la inseguridad y la delincuencia son el problema más importante que les afecta, seguido del bajo poder adquisitivo de la población. Esta misma fuente estima que en 2017 el $33.7 \%$ de las unidades económicas fue víctima de algún delito, $30.6 \%$ del sector industrial y $29.3 \%$ del sector servicios. De manera más puntual, se contabilizaron 3.8 millones de delitos asociados a 1.5 millones de unidades económicas. Esto representa una tasa de concentración de 2.53 delitos por unidad económica solamente durante 2017.

De acuerdo con el INEGI (2018b) el costo monetario total a consecuencia de la inseguridad y el delito en unidades económicas representó un monto de 155.8 mil millones de pesos, es decir, $0.86 \%$ del PIB, lo que ha provocado que las empresas reporten: "a) dejar de manejar efectivo, b) reducción de horarios, c) cancelación de inversiones, o d) dejar de hacer negocios".

La falta de una política de prevención, sumada a la reforma penal, que inició su ejecución en 2016, lejos de haber creado las bases de una justicia más eficiente, ha creado un ambiente de impunidad que favorece al robo como elección de actividad por parte de

\footnotetext{
${ }^{21}$ Encuesta Nacional de Victimización de Empresas (ENVE) y Encuesta Nacional sobre Victimización y Percepción de Seguridad Pública (ENVIPE).
} 
jóvenes varones con baja educación. Y eso se debe a que la reforma penal ha implicado la presencia de mecanismos alternativos ${ }^{22}$ y arreglos reparatorios del daño que se aplican hasta en delitos graves, como portación de armas (De Gante, 2017). Todo ello ha alentado al crimen. De manera que si bien esta reforma buscó proteger las garantías y los derechos humanos de los presuntos delincuentes, en realidad ha creado un ambiente de creciente impunidad al restringir la prisión preventiva y por ello se ha convertido en un factor que ha impulsado la criminalidad.

Otro delito que también puede analizarse a través de nuestro enfoque es el que tiene que ver con los "delitos de cuello blanco" 23 debido a que los montos derivados de ese tipo de delitos y la protección jurídica a la que pueden acceder los individuos que los realizan cumplen con la condición de que el beneficio neto del robo supera los beneficios del empleo legal. Sin embargo, habría que precisar que este delito en particular se comete en condiciones de empleo y no necesariamente de CCO ni por el grupo de edad motivo de este estudio.

Estimamos un modelo BVAR y los resultados revelaron la existencia de un "círculo vicioso" dinámico conformado por la tasa de desempleo, tasa de Condiciones Críticas de Ocupación (CCO) y la tasa de robos. Parte de que un choque económico adverso que eleve el desempleo y/o CCO de la población objeto tiende a desatar una dinámica que auto reproduce negativamente las condiciones de ocupación, la actividad económica y el crimen. Esta evidencia que ha sido demostrada econométricamente, ilustra claramente los condicionantes socioeconómicos del robo y el fracaso de las políticas de combate frontal al delito y de justicia penal definidas desde la década pasada.

Una estrategia exitosa deberá considerar estos factores como ejes centrales. Factores preventivos de orden educativo, recreacional y de ingresos llevarán más tiempo en funcionar, debido al letargo que sufre la economía mexicana desde hace tres décadas y a que la tasa de desempleo de los jóvenes es el doble respecto de la total. De esta manera, si las expectativas de inclusión laboral, de mayores salarios y de trayectoria laboral se mejoran es posible que el robo deje de ser una opción para los jóvenes. Así políticas públicas de capacitación laboral tendrían efectos de reducción del delito, pero después de cierto tiempo de aplicarse exitosamente.

Sin embargo, también debe actuarse simultáneamente con políticas que sean muy eficientes en el corto plazo como capacitar y reforzar el sistema policiaco mexicano. Es importante apuntar que aunque la reforma penal tiene 10 años de promulgada, apenas tiene 3 años de haber sido implementada y que el actuar de la policía aún no es el adecuado ya que ha sido incapaz de realizar no sólo las detenciones en la forma en que establece el "debido proceso", sino de aportar las evidencias sólidas para sustentar la acusación. Al respecto, Aguirre (2018) menciona que uno de los principales problemas de la policía es la falta de capacitación.

Sin una imputación adecuada, como lo exige el actual sistema penal, no es posible exigir que la autoridad judicial inicie el proceso penal, con lo que el crimen queda impune, y los elementos policiacos no capacitados no pueden siquiera realizar una detención o una consignación, tal vez por miedo de violar alguna garantía y ser a su vez acusados, con lo cual pueden enfrentar problemas legales, sumado al hecho de que el robo no es un delito en el que aplique la prisión preventiva. Por ello, es muy probable que no actúen ante la presencia o la denuncia de robos.

Por otro lado, el hecho de que muchos criminales salen libres por incumplimiento del debido proceso desincentiva el quehacer policiaco. Es por ello que muchos policías prefieren no actuar y de esa manera aumenta la incidencia del delito. La defectuosa aplicación del proceso que marca el actual sistema penal ha convertido a los ministerios públicos y a las prisiones en oficinas con puerta giratoria.

\footnotetext{
${ }^{22}$ Como la mediación, negociación y conciliación entre las partes en conflicto.

${ }^{23}$ Se añadió este comentario a pedido expreso de un árbitro.
} 


\section{Referencias}

Aguirre, J. P. (2018). Déficit de policías en México, problema de ingobernabilidad. RICEG. Revista Internacional de Ciencias del Estado y de Gobierno, 1(3), 397-419.

Arévalo, M. (2016). Impunidad y variables económicas en México: 2010-2012. Tesina para obtener el grado de Lic. en Relaciones Internacionales. CIDE, México.

Arias, J., Azuara, O., Bernal, P., Heckman, J. J. Villarreal, C. (2010). Policies to Promote Growth and Economic Efficiency in Mexico (No. w16554). National Bureau of Economic Research.

Banco Mundial (2013). Jóvenes de México, autores y víctimas de la violencia. http://www . bancomundial . $\mathrm{org} / \mathrm{es} / \mathrm{news} /$ feature/2013/03/05/mexican-youth-authors-and-victims-of-violence. Revisado 15 octubre de 2018 .

Beccaria, C. (1764). On Crimes and Punishments. http://criminologytoday.com/beccaria.pdf. Revisado 15 octubre de 2018

Becker, G. S. (1976). The Economic Approach to Human Behavior: University of Chicago Press.

Canova, F. (2007). Methods for Applied Macroeconomic Research. Princeton University Press. Vol. 13.

Cantor, D. Land, K. C. (1985). Unemployment and Crime Rates in the Post-World War II United States: A Theoretical and Empirical Analysis. American Sociological Review, 317-332.

Cid, J. (2007). ¿Es la prisión criminógena? Un análisis comparativo de reincidencia entre la pena de prisión y la suspensión de la pena, Revista de Derecho Penal y Criminología, 2da Época, no. 19. 427-456.

De Gante, A. H. (2017). Reforma Penal en México: ¿Mayor seguridad o mayor violencia? Revista de Derecho: Publicación de la Facultad de Derecho de la Universidad Católica de Uruguay, (16), 137163.

Dornbusch, R., Fischer, S. Startz, R. (2004). Macroeconomics. 9th ed. Mc Graw Hill.

ENOE (2018). Consulta interactiva de datos. http://www.inegi.org. $\mathrm{mx} / \mathrm{est} / \mathrm{lista}_{c} u b o s /$ consulta.asp $? \mathrm{p}=$ encuec $=4$.Revisadoel20demayode2018.

Fajnzylber, P., Lederman, D. Loayza, N. (1998). Determinants of Crime Rates in Latin America and the World, an Empirical Assessment. World Bank

Freeman, R. B. (1999). The Economics of Crime. Handbook of Labor Economics, 3, 3529-3571.

Gómez, V. y Merino J. (2012). "Ninis" y la violencia en México: ¿nada mejor que hacer nada o nada mejor que esperar? En Aguilar, J. (Coordinador), Las bases sociales del crimen organizado y la violencia en México. CIES y SSP. México, 133-185.

INEGI. (2018a). Encuesta Nacional de Victimización y Percepción sobre la Seguridad Pública. http:// www.beta.inegi.org.mx/contenidos/proyectos/enchogares/regulares/envipe/2018/doc/envipe 2018_presentacion_nacional.pdf. Revisado 16 de octubre de 2018.

INEGI (2018b). Resultados de la cuarta edición de la Encuesta Nacional de Victimización de Empresas (ENVE) http://www.beta.inegi.org.mx/contenidos/saladeprensa/boletines/2018/EstSegPub/ ENVE2018.pdf. Revisado 26 de octubre de 2018.

Infante, S., Alvarado, L. y Vera, J. (2010). Políticas de equidad, educación y delincuencia juvenil en México. Buenos Aires. Ibero-American Education Congress. Argentina.

Islas, A. y Cortez, W. (2018). ¿Puede el sector informal afectar a la relación entre desempleo y producción? Un análisis del caso de México. Revista de la CEPAL. No 126, diciembre.

Kadiyala, K. R. Karlsson, S. (1997). Numerical Methods for Estimation and Inference in Bayesian VAR models. Journal of Applied Econometrics, 99-132.

Litterman, R. (1984). Specifying Vector Autoregressions for Macroeconomic Forecasting, Federal Reserve Bank of Minneapolis, Staff Report no. 92

Loría, E., Ramírez, E. y Salas, E. (2015). La Ley de Okun y la flexibilidad laboral en México: un análisis de cointegración, 1997Q3-2014Q1. Contaduría y Administración, 60(3), 631-650.

Lu, Q., Zhang, Z., Chai, J. Zhu, Q. (2013). The Analysis of the Market of Coal Consumption in Shaanxi Province Based on the BVAR model, in Business Intelligence and Financial Engineering (BIFE), 2013 Sixth International Conference on IEEE. 490-494).

Mas-Colell, A., Whinston, M. D. Green, J. R. (2006). Microeconomic Theory. New York: Oxford University Press

Mendoza, E. (2013). Introducción, en Mendoza, E. (Coordinador), La crisis financiera internacional: efectos sectoriales en México y en su frontera norte. El Colegio de la Frontera Norte. México. 11-27.

Moreno, C. y Toledo, A. (2017). Los menores de edad en la delincuencia organizada en México. Letras Jurídicas, 15(15)

Molzahn, C., Ríos, V. Shirk, D. (2012). Drug Violence in Mexico. Data and Analysis Through 2011. San Diego: Trans-Border Institute.

Obando, N. y Ruiz, C. (2007). Determinantes socioeconómicos de la delincuencia: una primera aproximación a nivel provincial. Informe final. Consorcio de investigación económica y social. http://www . cies.org.pe/sites/default/files/investigaciones/determinantes-socioeconomicos-de-la-de. lincuencia-una-primera-aproximacion-al-problema-a-nivel-provincial .pdf. Consultado 18 diciembre 2017. 
Okun, A. (1962). Potential GNP: Its Measurement and Significance. American Statistical Association, Proceedings of the Business and Economic Statistics Section, 98-104.

Pesaran, H. H. Shin, Y. (1998). Generalized Impulse Response Analysis in Linear Multivariate Models. Economics Letters, 58(1), 17-29.

PNUD. (2013). Informe Regional de Desarrollo Humano 2013-2014. Nueva York: ONU. http://www. latinamerica. undp.org/content/dam/rblac/img/IDH/IDH-AL\%20Inf orme\%20completo.pdf. Revisado 26 de julio de 2018.

Prachowny, M. F. (1993). Okun's Law: Theoretical Foundations and Revised Estimates. The Review of Economics and Statistics, 331-336.

Quilis, E. M. (2002). Modelos BVAR: especificación, estimación e inferencia. Instituto de Estudios Fiscales. España.

Ramírez-de-Garay, D. y Román, M. P. D. (2017). Los efectos de la política de prevención del crimen y la violencia en México. Revista Cidob D'Afers Internacionals, 101-128

Roemer, A. (2001). Economía del crimen. Ed. Limusa. México.

Singh, K. Xie, M. (2008). Bootstrap: a Statistical Method. Unpublished Manuscript, Rutgers University, USA. Retrieved from http://www. stat. rutgers. edu/home/mxie/RCPapers/bootstrap. pdf. Consulted December 18, 2017.

SNSP (2019). Secretariado Ejecutivo Nacional de Seguridad Pública. http://secretariadoejecutivo. gob.mx/index.php. Revisado 26 de septiembre de 2018.

STPS (2017). Glosario de términos laborales. http://www.stps.gob.mx/gobmx/estadisticas/Glosario/ glosario.htm. Revisado 18 diciembre 2017.

Thornberry, T. P. Christenson, R. L. (1984). Unemployment and Criminal Involvement: An investigation of reciprocal causal structures. American Sociological Review, 398-411.

Urrusti, S (2012). La violencia como consecuencia de la falta de coordinación política, en Aguilar, J (Coordinador). Las bases sociales del crimen organizado y la violencia en México (337-364). CIES y SSP. México.

Varella, J. (2014). Juventud, violencia y delincuencia desde una mirada de la prevención social del delito. Revista Observatorio de la Juventud. (29), 39-54

Zuluaga, D., Sánchez, F. y Chegwin, V. (2018). Empleo, Violencia y Oportunidades para los Jóvenes. Evidencia para América Latina y el Caribe. Documentos CEDE. No.14.

Zuur, A. F., Leno, E. N. Elphick, C. S. (2010). A Protocol for Data Exploration to Avoid Common Statistical Problems. Methods in Ecology and Evolution, 1(1), 3-14. 